\title{
Preface: Advanced Thermal Strategies in Cancer Therapy and Diagnostics
}

Mild hyperthermia and thermal ablation have been adopted as minimally invasive strategies for the treatment of malignant diseases. These treatments use thermal energy sources such as radiofrequency (RF), microwave, laser, and high-intensity focused sonography. Planning and control of thermal treatments is essential to avoid formation of thermal lesions and overheating of surrounding healthy tissues.

The current issue of Critical Reviews ${ }^{\mathrm{TM}}$ in Biomedical Engineering, "Advanced Thermal Strategies in Cancer Therapy and Diagnostics," examines recent research in planning and monitoring of thermal ablation therapy. It also presents noninvasive thermal techniques developed to improve the accuracy in diagnostics of malignancy as well as research on thermal monitoring of tumor response during radiation and chemotherapy treatments.

Various ultrasound-based methods have been studied in the field of noninvasive thermal therapy monitoring for the mild hyperthermia. However, at high temperatures, which are the case for thermal ablation, especially when the tissue is coagulated, ultrasound temperature monitoring becomes problematic. "Microwave-Induced Thermal Lesion Detection via Ultrasonic Scatterer Center Frequency Analysis with Autoregressive Cepstrum" by Dr. Sheng et al. ${ }^{1}$ presents a novel methodology that can be used for noninvasive monitoring of the microwave ablation therapy. To prevent complications while ensuring treatment efficacy, the authors proposed a new method for microwave-induced thermal lesion detection using the autoregressive spectrum analysis of ultrasonic backscattered signals.

Planning and control of thermal treatments is enhanced by mathematical modeling that allows to predict and analyze the temperatures during treatment. The complex phenomena that need to be modeled involve interaction of a heat source with human tissues, blood perfusion, and metabolic heat generation. Additional complexity arises from uncertainty of thermophysical parameters that need to be taken into account for an accurate planning and control of hyperthermia and thermal ablation. In "Thermal Characterization of an Ex Vivo Tissue," Dr. Orlande and his team estimated thermophysical properties of an ex vivo tissue through solution of inverse problems using the Markov chain Monte Carlo method. ${ }^{2}$ Estimated temperatures were confirmed by comparison with experimental measurements. In addition, the approximate Bayesian computation algorithm was applied to calculate the parameters of an Arrhenius thermal decomposition. This allowed to estimate thermal damage parameters of bovine muscle heated by thermal radiation.

In "Numerical Simulation of Effects of Bioheat Transfer Characteristics of Malignant Melanoma on Thermal Conductivity Measurements," Dr. Okabe et al. discuss modality for thermally diagnosing a malignant melanoma via thermal conductivity measurements. ${ }^{3}$ This modality is based on the use of a guard-heated thermistor probe, which is a penshaped device used for measuring the skin-surface temperature and the effective thermal conductivity of the skin. In this work, to explain the clinical data obtained for the patients with stage IV invasive melanoma, the authors numerically investigated the cause of differences in the measured effective thermal conductivity.

Radiation recall dermatitis is an acute inflammatory reaction confined to previously irradiated areas that can be triggered when chemotherapy agents are administered after radiotherapy. In "Thermal Monitoring of Tumor and Tissue State During Radiation Therapy - A Complex Case of Radiation Recall," Dr. Gannot and his team investigated the feasibility of using thermography as a tool to predict the response of normal breast tissue and skin to radiation therapy and the risk of developing radiation recall dermatitis. ${ }^{4}$ They demonstrated the differences between thermographic radiation skin response in adjuvant radiation to the breast and in breasts with viable tumor, as well as a special case of radiation recall phenomena and its characteristics. 


\section{REFERENCES}

1. Sheng L, Rao W, Zho Z, Wu S, Ma G. Microwave-induced thermal lesion detection via ultrasonic scatterer center frequency analysis with autoregressive cepstrum. Crit Rev Biomed Eng. 2020.

2. Loiola BR, Abreu LAS, Orlande HRB. Thermal characterization of an ex-vivo tissue. Crit Rev Biomed Eng. 2020.

3. Okabe T, Okajima J, Fujimura T, Aiba S, Maruyama S. Numerical simulation of effects of bioheat transfer characteristics of malignant melanoma on thermal conductivity measurements. Crit Rev Biomed Eng. 2020.

4. Ben-David MA, Hoffer O, Kirshenabum D, Katz E,
Alezra D, Symon Z, Rabin T, Zimmer Y, Kelson I, Gannot I. Thermal monitoring of tumor and tissue state during radiation therapy - a complex case of radiation recall. Crit Rev Biomed Eng. 2020.

\section{Guest Editor:}

\section{Victoria Timchenko}

School of Mechanical and Manufacturing Engineering

The University of New South Wales

Sydney, Australia 